\title{
Simple experimental test to distinguish extraction and injection barriers at the electrodes of (organic) solar cells with S-shaped current-voltage characteristics
}

Wolfgang Tress and Olle Inganäs

\section{Linköping University Post Print}

\section{Tweet}

N.B.: When citing this work, cite the original article.

Original Publication:

Wolfgang Tress and Olle Inganäs, Simple experimental test to distinguish extraction and injection barriers at the electrodes of (organic) solar cells with S-shaped current-voltage characteristics, 2013, Solar Energy Materials and Solar Cells, (117), SI, 599-603.

http://dx.doi.org/10.1016/j.solmat.2013.07.014

Copyright: Elsevier

http://www.elsevier.com/

Postprint available at: Linköping University Electronic Press

http://urn.kb.se/resolve?urn=urn:nbn:se:liu:diva-100304 


\title{
Simple experimental test to distinguish extraction and injection barriers at the electrodes of (organic) solar cells with S-shaped current-voltage characteristics
}

\author{
Wolfgang Tress ${ }^{\mathrm{a}, \mathrm{b}}$, Olle Inganäs ${ }^{\mathrm{a}}$ \\ ${ }^{a}$ Biomolecular and Organic Electronics, IFM, Linköping University, 58183 Linköping, Sweden \\ ${ }^{b}$ Institut für Angewandte Photophysik, Technische Universität Dresden, George-Bähr-Str. 1, 01069 Dresden, Germany
}

\begin{abstract}
Adjusting the work function of the two electrodes to the energy levels of the intrinsic active materials of an organic solar cell is crucial for a good device performance. Often, injection barriers (in combination with selective contacts blocking one charge carrier species) caused by a misaligned metal work function or extraction barriers resulting from insulating intentional or unintentional interlayers between metal and active layers, result in a decrease in fill factor seen in the extreme case in S-shaped current-voltage $(J-V)$ characteristics. To avoid this S-kink, it is essential to identify its origin, desirably applying a simple experimental method. We propose an approach based on analyses of current-voltage data as a function of illumination intensity. A normalization of the $J$ - $V$ curves at the saturated photocurrent reveals distinctive features for each type of barrier. We apply the method to planar heterojunction small-molecule and bulk heterojunction polymer solar cells with oxidized metal electrode or plasma-treated active layer and explain the theory with a drift-diffusion model.
\end{abstract}

Keywords: organic solar cell; S-shape; barrier; electrode; drift-diffusion model; plasma-treatment

The development in organic photovoltaics is mainly driven by new materials $[1,2]$. These materials require tailored interfaces to the electrodes. Furthermore, at the crossover from laboratory to large-scale fabrication, organic photovoltaics are increasingly affected by processing, stability, economic and environmental constraints regarding the employment of electrode materials and the sequence of layer deposition $[3,4]$. Therefore, new architectures arise which show the need of a better control of interface properties to achieve a similar performance as devices in the standard architecture [5]. However, often a decrease of solar cell performance is observed when employing alternative electrodes. Mostly the open-circuit voltage $\left(V_{\mathrm{oc}}\right)$ or the fill factor $(\mathrm{FF})$ are negatively affected. The low FF often comes along with distorted current-voltage $(J-V)$ curves in the extreme case showing inflection points close to $V_{\text {oc }}$ (so-called S-kink) $[6,7,8,9]$.

In our previous work we explained the factors giving rise to S-kinks $[10,11,12]$. There, we identified energy barriers at the electrodes as the main reason. Other studies investigated the role of energy barriers due to misaligned electrode work functions as well[13, 14, 15, 16]. Using tailored experiments and accompanying simulations we demonstrated a correlation of the barrier height and the strength of the S-kink. Combining materials with different energy levels, we realized injection and extraction barriers for charge carriers, both giving rise to S-kinks in the $J-V$ curves of planar heterojunction solar cells [10].

Email address: woltr@ifm.liu.se (Wolfgang Tress)
These S-kinks look similar independent of the type of barrier. However, this strategy of intentionally incorporating energy barriers is neither a common nor a desired approach when designing and fabricating solar cells. Commonly, an unwanted S-kink is observed during the characterization of a novel device. Therefore, the reasons for the S-kink are initially unknown and it would be highly desired to extract them directly from the current-voltage characteristics. This knowledge would allow a fast and systematic improvement of the device performance, as the correct measure will depend on the kind of problem, i.e. type of barrier, present: An extraction barrier implies that charges cannot leave the device because the interface (layer) is not well-conducting or shows a huge energy barrier for charges when being extracted. This issue requires making this material more conducting and thinner, or completely replace it. Injection barriers, however, mean that the work function of a selective electrode is not sufficiently high (low) to match the HOMO (LUMO) of the donor (acceptor). In this case, a modification of its work function by introducing a dipole layer will increase the fill factor. Thus, the correct measure to take depends on the knowledge of the factor causing the S-kink.

Here, we propose a simple experimental approach to identify the correct type of barrier. This method only requires $J-V$ measurements in an illumination intensity range of about 0.001 to 1 sun. We will show that a normalization of the intensity dependent current-voltage data at a reverse bias point directly discloses the reason for the S-kink. We elaborate this method on small-molecule so- 
lar cells where the barriers are intentionally introduced and known in type and magnitude. A generalization and explanation is done using drift-diffusion simulations. Furthermore, we show the power of this approach by applying it to inverted polymer-fullerene solar cells with interface modifications.

We first discuss the case of an extraction barrier. Charge carriers, in our case, holes experience an energy barrier for extraction, which might result from an insulating layer between metal electrode and active layer. In the device whose data is shown in Fig. 1(a), the extraction barrier is intentionally created by a deeper lying highest molecular orbital (HOMO) of the hole-transport layer (HTL) compared to the donor [see inset of Fig. 1(a)]. As discussed in Ref. [10] this energy barrier can lead to Sshaped $J-V$ curves. This S-kink is present for illumination intensities down to 0.005 suns, as shown in Fig. 1(a) where the $J$ - $V$ data recorded under varied illumination intensities is normalized at reverse bias. The voltage (here $-0.5 \mathrm{~V}$ ) should be chosen in a way that the normalized $J-V$ curves coincide below this value. Ideally from this point on the photocurrent should be saturated and the choice of the exact voltage value is insignificant. In real devices a trade-off has to be found to decrease effects of the dark curve (e.g. break-through) which governs the current of the lowest intensity curve in Fig. 1(a). To reduce the effects of the dark current, one might plot normalized photocurrent data as difference between the $J$ - $V$ curves under illumination and in dark. The normalization allows for a comparison of the strength of the S-kink which is pronounced for the highest intensities and decreases gradually until it vanishes for low intensities. This behavior leads to points of intersection of the normalized $J$ - $V$ curves as $V_{\text {oc }}$ increases with light intensity. These points of intersection are characteristic for the presence of an extraction barrier, as visualized with simulation data in Fig. 2(a).

This trend in the strength of the S-kink can be explained when looking at the reason for the S-kink visualized in Fig. 3(a). There, spatial profiles of charge density and electric field are plotted showing that photogenerated holes pile up in front of the barrier at the interface donor/HTL. This charge partly screens the field in the device which originates from the built-in potential $\left(V_{\mathrm{bi}}\right)$ at $0 \mathrm{~V}$ applied bias. For applied bias voltages, the field in the device is given by a superposition of both potentials. Due to the screening effect, most of the potential drops over the intrinsic HTL which behaves like the dielectrics of a capacitor. The enhanced field in the HTL gives rise to a reduced field in donor and acceptor and in particular at the D/A interface. This lower field leads to a higher probability of recombination at the $\mathrm{D} / \mathrm{A}$ interface. Therefore, the photocurrent at a fixed applied bias is reduced compared to a solar cell without barrier at the same applied bias. That is why the $J-V$ curve shows an S-kink. The increased strength of the S-kink with illumination intensity is already included in this explanation. The higher the intensity, the more charges are photogenerated which can pile-up at the barrier [Fig. 3(a)]. Therefore, at a fixed voltage point (shown here $0 \mathrm{~V}$ ) the field increases in the HTL and decreases at the D/A interface with enhanced light intensity. Thus, a higher share of the generated charges will recombine. Normalizing the $J-V$ curve to the saturated reverse photocurrent gives rise to plots like those in Figs. 1(a) and 2(a).

Now we turn focus to devices with an injection barrier, which arises from a work function of the electrode within the energy gap of a semiconductor or in our case a HOMO of the HTL lying higher than the HOMO of the donor. We again plot normalized $J-V$ data [Fig. 1(b)]. At first glance the data looks similar to the case of an extraction barrier. However, there is a distinct difference: a point of intersection of the normalized $J$ - $V$ curves cannot be observed. Several curves for intensities larger than 0.1 suns coincide until a point close to $V_{\text {oc }}$. This effect is reproduced in simulation data shown in Fig. 2(b). The reason is related to the origin of the S-kink in the presence of an injection barrier. An injection barrier implies a low built-in potential $V_{\mathrm{bi}}$ compared to the $\mathrm{HOMO}_{\text {donor }}-\mathrm{LUMO}_{\text {acceptor }}$ difference. This means that in case of a selective device architecture (selective contacts or a planar heterojunction), a $V_{\text {oc }}$ higher than $V_{\text {bi }}$ is possible. In such a case the current close to $V_{\text {oc }}$ is completely diffusion-driven against the field. This competition of diffusion and drift current is seen in an S-shape. As $V_{\text {oc }}$ decreases with reduced light intensity, a threshold intensity will be reached where $V_{\text {oc }}$ becomes smaller than $V_{\mathrm{bi}}$. Then, the S-kink vanishes. In the data of Fig. 1(b), this is the case for intensities lower than 0.005 suns. For higher intensities the S-kink is clearly visible. However, charge carrier density and electric field profiles [Fig. 3(b)] do not change significantly with intensity. The simulations show a high concentration of holes in the intrinsic HTL resulting from a diffusion of holes from the p-doped HTL where a negative space charge remains. Due to the injection barrier at the HTL/donor interface, the holes are inhibited from diffusing into the donor. This space charge gives rise to a high electric field at the junction p-doped HTL/intrinsic-HTL comparable to a conventional $\mathrm{p}-\mathrm{n}$ junction. Note that the field at the $\mathrm{D} / \mathrm{A}$ interface is negative due to the applied bias of $0.8 \mathrm{~V}$ and the low $V_{\mathrm{bi}}$. As the field distribution results from dark carriers and does not significantly change with light intensity, the shape of the $J-V$ curve remains mostly unaffected. That is why all $J$ - $V$ curves in Figs. 2(b) and 1(b) coincide as soon as the intensity is large enough to provide a $V_{\text {oc larger than }} V_{\text {bi }}$. They deviate only close to $V_{\text {oc }}$ to account for the diffusion current and the increased $V_{\mathrm{oc}}$ with intensity. The $J-V$ curve at very high intensity in Fig. 2 is an exception. The reason is that at very high intensities, bimolecular recombination at the interface dominates the $J$ - $V$ curve besides the barrier and leads to a decrease in fill factor.

Before applying the presented findings to polymer:fullerene bulk heterojunction systems, we want to mention that other slightly more sophisticated experimen- 

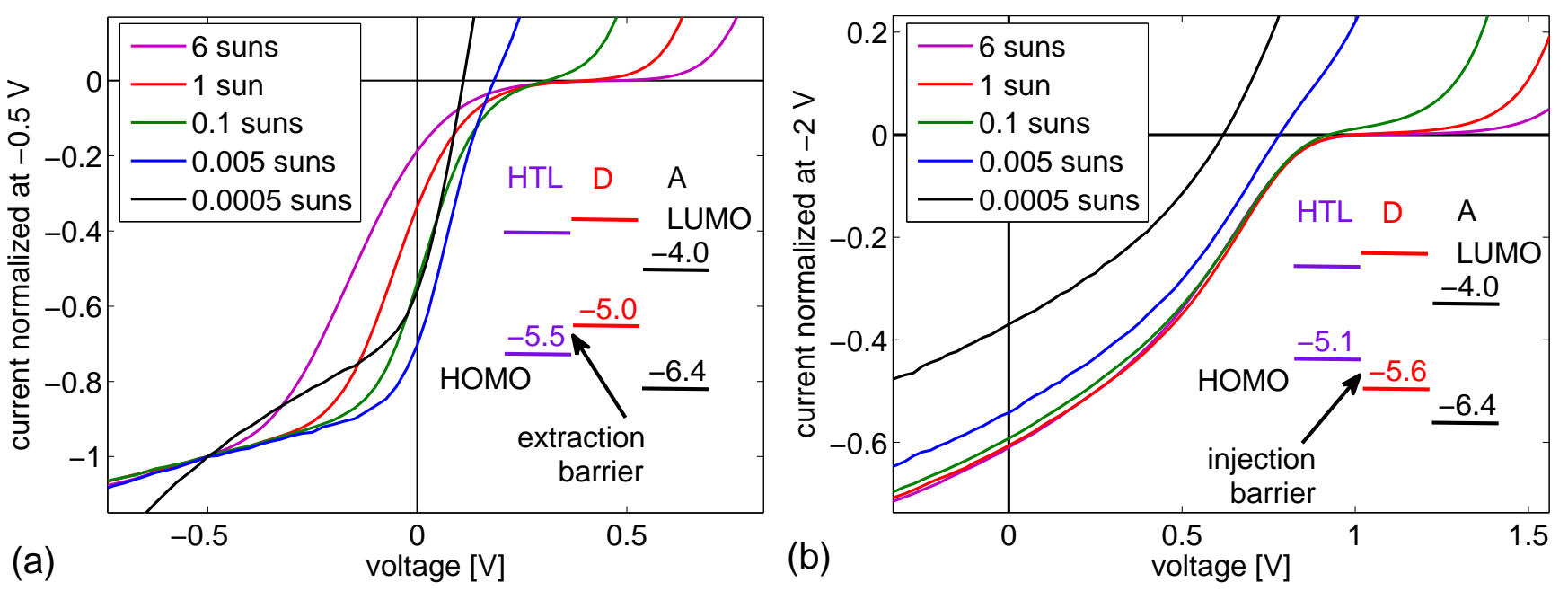

Figure 1: Experimental data: Normalized $J-V$ curves for a series of illumination intensities, here given in quantities of 1 sun, which was set to $150 \mathrm{~mW} / \mathrm{cm}^{2}$ monitored by a calibrated silicon reference diode. The differences in forward direction (current $>0$ ) are mainly an artifact resulting from the normalization. The insets show the energy levels (in $\mathrm{eV}$ ) of the materials employed, where the energy of the highest occupied molecular orbital (HOMO) is defined via the ionization potential (IP) measured by photoelectron spectroscopy at thin films. The LUMO indicates the energy of the lowest unoccupied molecular orbital. HTL means hole-transport layer, D donor, and A acceptor. (a) Device with extraction barrier for holes: ITO/p-doped $\alpha$-NPB $(20 \mathrm{~nm}) / \alpha$-NPB $\left(8 \mathrm{~nm}, \mathrm{HOMO}_{\mathrm{IP}} \approx-5.5 \mathrm{eV}\right) / \mathrm{ZnPc}(8 \mathrm{~nm}, \mathrm{HOMO}$ IP $\approx-5.0 \mathrm{eV}) /$ $\mathrm{C}_{60}(40 \mathrm{~nm}) / \mathrm{BPhen}(6 \mathrm{~nm}) / \mathrm{Al}(100 \mathrm{~nm})$. (b) Device with injection barrier for holes: ITO/ p-doped MeO-TPD $(20 \mathrm{~nm}) / \mathrm{MeO}-\mathrm{TPD}(8 \mathrm{~nm}$, $\left.\mathrm{HOMO}_{\mathrm{IP}} \approx-5.1 \mathrm{eV}\right) / \mathrm{BPAPF}\left(8 \mathrm{~nm}, \mathrm{HOMO}_{\mathrm{IP}} \approx-5.6 \mathrm{eV}\right) / \mathrm{C}_{60}(40 \mathrm{~nm}) / \mathrm{BPhen}(6 \mathrm{~nm}) / \mathrm{Al}(100 \mathrm{~nm})$. Details on the devices and materials can be found in Refs. $[10,17]$.
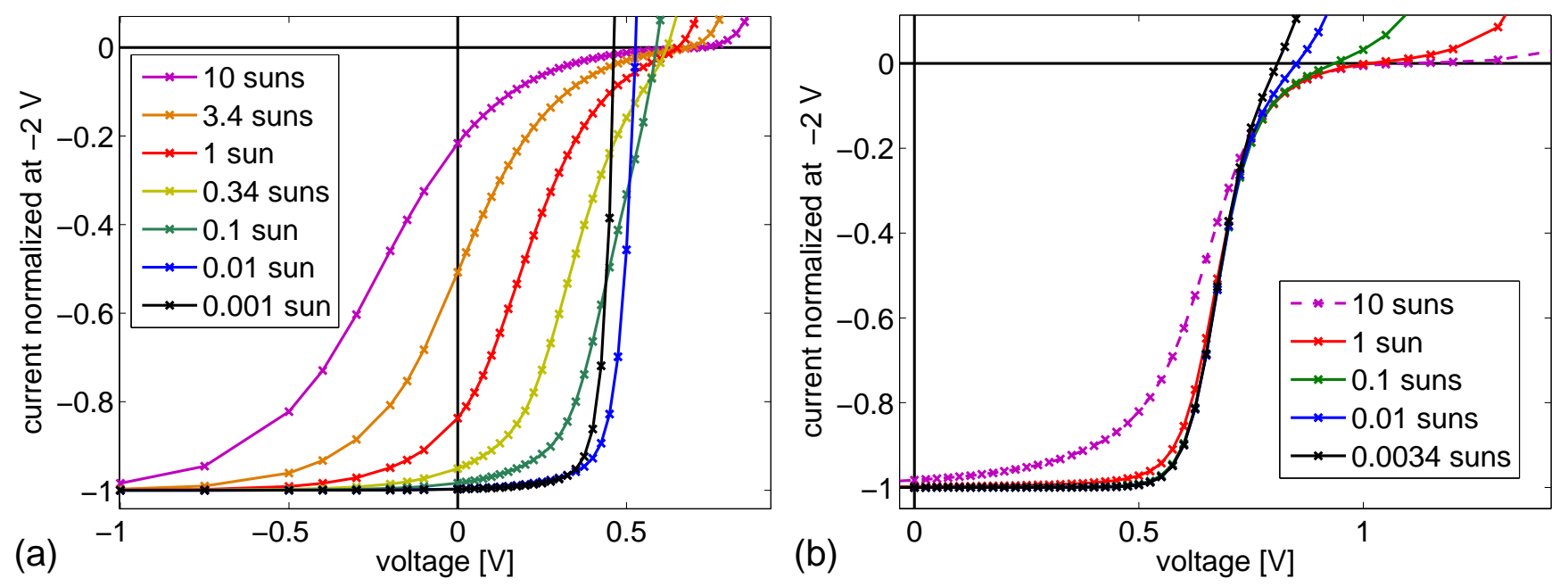

Figure 2: Simulation data: Normalized $J$ - $V$ curves obtained from a drift-diffusion simulation of the stacks mentioned in Fig. 1, assuming thermally activated jumps over the interface barriers including a field-dependent lowering term with a jump distance of $1 \mathrm{~nm}[10,17]$. (a) extraction barrier of $0.3 \mathrm{eV}$, (b) injection barrier of $0.3 \mathrm{eV}$. 

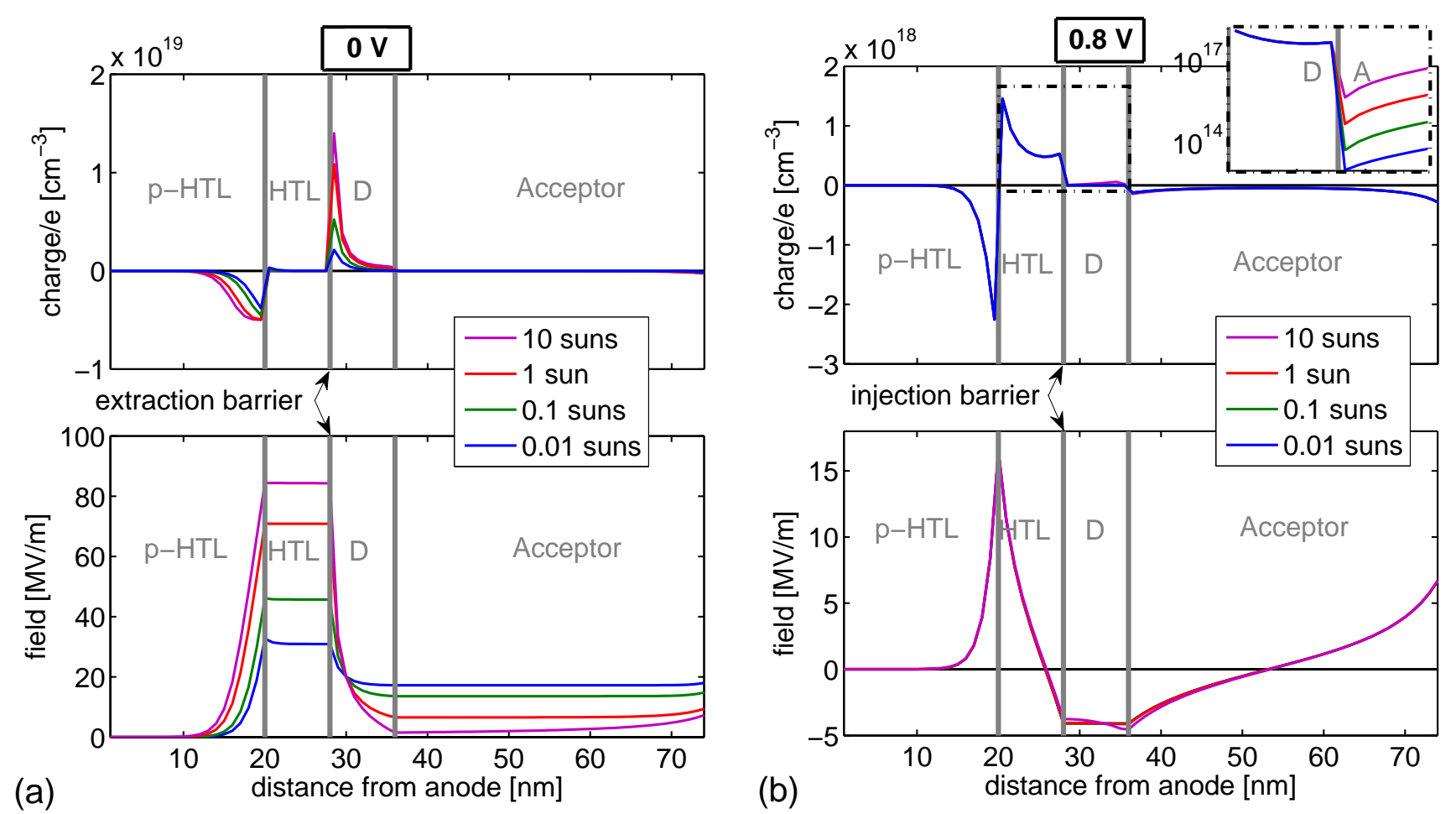

Figure 3: Simulated spatial distribution of space charge divided by elementary charge $e$ and electric field within the device stack at an applied bias voltage in the S-kink regime. The space charge mainly results from localized anions in the p-doped HTL, holes in the intrinsic HTL and donor, and electrons in the acceptor. A field larger than 0 indicates the desired direction for charge extraction, i.e. a field driving electrons to the cathode and holes to the anode. (a) Extraction barrier device at $0 \mathrm{~V}$. The higher the illumination intensity the more charges pile up at the HTL/donor interface which leads to a reduction of the field at the D/A interface. (b) Injection barrier device at $0.8 \mathrm{~V}$. As photogenerated charges do not pile up, the field is almost independent of illumination intensity. As the field is negative at the D/A interface, charge extraction is driven by diffusion against the field. The inset shows that the charge carrier density at the D/A interface and therefore the diffusion gradient and in turn $V_{\mathrm{oc}}$ increase with light intensity.
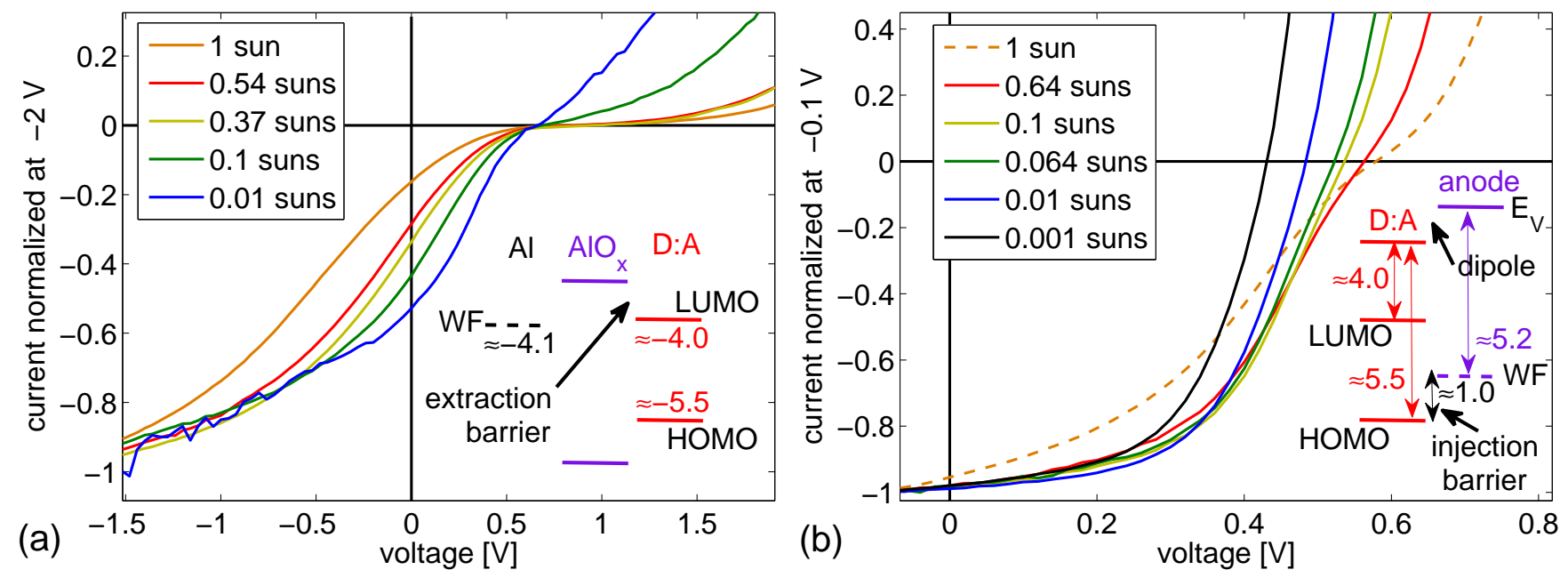

Figure 4: Experimental data: Normalized $J$ - $V$ data of bulk heterojunction solar cells based on poly[2,3-bis-(3-octyloxyphenyl)qui-noxaline5,8-diyl-alt-thiophene-2,5-diyl] (TQ1) : [6,6]-phenyl-C71-butyric acid methyl ester ([70]PCBM). (a) Al (80 nm) / TQ1:PCBM (80 nm)/ PEDOT:PSS PH1000. The data show characteristics of an extraction barrier probably caused by an oxidation of the Al electrode as sketched in the inset (energy levels in eV). (b) Al $(80 \mathrm{~nm}) / \mathrm{Ti}(2 \mathrm{~nm}) /$ PFPA-1 / TQ1:PCBM (80 nm, plasma-treated)/ PEDOT:PSS PH1000. The data indicate that the (air) plasma treatment of the surface of the active material gives rise to a dipole resulting in an injection barrier as sketched in the inset, where $E_{\mathrm{V}}$ denotes the vacuum level and WF the work function of the electrode.

tal approaches can be applied to distinguish between injection and extraction barrier: A variation of layer thick- nesses can directly reveal the kind of barrier as well [17]. If the donor layer thickness is increased, the shape of the 
$J-V$ curve will be affected for injection barriers only. In contrary, an increased HTL thickness enhances the effect of the extraction barrier and thus the strength of the Skink. The reason is that it is always the layer behind (in direction of current flow) the interface with the barrier that influences the probability of charge carriers crossing the barrier. As discussed, charge carriers pile up in front of a barrier and lead to a space charge that makes the layer in front of the barrier relatively field-free. The voltage drops mainly over the layer behind the barrier and the electric field at the barrier, which defines the charge transport over a given barrier, is higher the thinner this layer. Also transient photocurrent measurements are capable of giving insights into the driving forces for charge-carrier extraction [18].

In the following, we use the solar cell stack, materials, and preparation procedures detailedly described in Refs. [19, 20]. In Ref. [20] an inverted geometry[21] is presented with the layer sequence glass substrate/metal cathode $(80 \mathrm{~nm}) / \mathrm{TQ1}: \mathrm{PCBM}$ $(80 \mathrm{~nm}) / \mathrm{PEDOT}$ anode. Whereas the reference solar cell with $\mathrm{Ti} / \mathrm{Al}(80 \mathrm{~nm}) / \mathrm{Ti}(2 \mathrm{~nm})$ cathode shows good performance (fill factor of 0.53) [20], the device without the titanium interlayer exhibits a huge S-kink despite similar work functions[22] of $\mathrm{Al}$ and $\mathrm{Ti}$ [Fig.4(a)]. Although prepared in a glove box (oxygen content lower than $5 \mathrm{ppm}$ ), the Al surface is suspected to be oxidized[21]. Normalized $J-V$ data for varied illumination intensities are shown in Fig. 4(a) and visualize that the S-kink gets more pronounced for higher intensities. This implies a point of intersection of the curves which can also be seen. Comparing with Fig. 2 we identify this behavior as caused by an extraction barrier. This means that indeed an insulating layer (most probably $\mathrm{AlO}_{\mathrm{x}}$ ) is formed. This material is insulating due to its high bandgap and energy barriers for charge injection into $\mathrm{AlO}_{\mathrm{x}}$ [23]. Only for very high negative bias voltages, charges can pass (probably via tunneling) through this thin insulating layer. The formation of an aluminum oxide layer upon exposure to oxygen or water vapor was reported in literature [24, 25]. The oxidation of the $\mathrm{Al}$ electrode and organic molecules at the interface was also reported in a detailed study in Ref. [26], showing that $\mathrm{Ti}$ gives an ohmic contact. We observe that $2 \mathrm{~nm}$ of $\mathrm{Ti}$ avoid the oxidation of $\mathrm{Al}$ completely. A possibly formed $\mathrm{TiO}_{\mathrm{x}}$ layer shows favorable states for electron transport[27].

As second example we select the same stack on a $\mathrm{Ti} / \mathrm{Al}$ $(80 \mathrm{~nm}) / \mathrm{Ti}(2 \mathrm{~nm}) /$ PFPA-1[20] electrode and perform an air plasma treatment on the active layer before depositing PEDOT. The motivation for the plasma treatment is to make the layer more hydrophilic such that the adhesion of the polar (aqueous) PEDOT:PSS can be increased, as delamination of this layer is a serious problem. We could show via a scotch test that adhesion is significantly enhanced and the device performance hardly changed upon plasma treatment[28]. However, as seen in Fig. 4(b) a too strong plasma treatment induces an S-kink. This might either be caused by a damage of the topmost molecules making them insulating (extraction barrier) or by a dipole introduced (injection barrier). Examining the normalized data reveals the characteristics of an injection barrier (cf. Fig. 2). Thus, the plasma treatment, making the surface more polar by attaching oxygen, also introduces a dipole, effectively decreasing the work function of PEDOT and thus causing a stronger misalignment of the work function of PEDOT:PSS to the HOMO of TQ1. A detailed photoelectron-spectroscopy study indeed identified a dipole at the active layer surface after plasma treatment[28]. Furthermore, the plasma-treated interface seems to be quite selective, which means polymer-rich and/or electron-blocking. Otherwise, an S-kink cannot be observed in case of an injection barrier.

In conclusion we have proposed a simple experimental test to distinguish between injection and extraction barriers at the electrodes. It is based on an analysis of normalized $J-V$ data recorded at varied illumination intensities. We elaborated this method for small-molecule solar cells with misaligned hole transport layers, explained it with drift-diffusion simulations, and successfully applied it to inverted polymer:PCBM solar cells. Quickly identifying the reason for S-kinks by this method will allow for a faster and tailored optimization of organic and other types of multilayer thin-film solar cells.

The authors thank the BMBF (OPEG, grant no. 13N9720) for financial support. WT kindly acknowledges funding from the Reiner Lemoine foundation. We thank Moritz Riede (IAPP, TU Dresden) for collaboration and discussion. Research in organic photovoltaics in Linköping is supported by the Science Council (V), the Swedish Energy Agency, and the Knut and Alice Wallenberg Foundation.

\section{References}

[1] J. Nelson, Polymer:fullerene bulk heterojunction solar cells, Materials Today 14 (2011) 462-470.

[2] G. Li, R. Zhu, Y. Yang, Polymer solar cells, Nature Photonics 6 (2012) 153-161.

[3] F. C. Krebs, T. Tromholt, M. Jø rgensen, Upscaling of polymer solar cell fabrication using full roll-to-roll processing., Nanoscale 2 (2010) 873-86.

[4] N. Espinosa, R. García-Valverde, A. Urbina, F. C. Krebs, A life cycle analysis of polymer solar cell modules prepared using roll-to-roll methods under ambient conditions, Solar Energy Materials and Solar Cells 95 (2011) 1293-1302.

[5] F. C. Krebs, S. a. Gevorgyan, J. Alstrup, A roll-to-roll process to flexible polymer solar cells: model studies, manufacture and operational stability studies, Journal of Materials Chemistry 19 (2009) 5442

[6] J. Meiss, M. K. Riede, K. Leo, Towards efficient tin-doped indium oxide (ITO)-free inverted organic solar cells using metal cathodes, Applied Physics Letters 94 (2009) 013303.

[7] C. S. Kim, S. S. Lee, E. D. Gomez, J. B. Kim, Y.-L. Loo, Transient photovoltaic behavior of air-stable, inverted organic solar cells with solution-processed electron transport layer, Applied Physics Letters 94 (2009) 113302.

[8] T. Kuwabara, Y. Kawahara, T. Yamaguchi, K. Takahashi, Characterization of inverted-type organic solar cells with a $\mathrm{ZnO}$ 
layer as the electron collection electrode by ac impedance spectroscopy., ACS applied materials \& interfaces 1 (2009) 2107-10.

[9] T. Stubhan, M. Salinas, A. Ebel, F. C. Krebs, A. Hirsch, M. Halik, C. J. Brabec, Increasing the Fill Factor of Inverted P3HT:PCBM Solar Cells Through Surface Modification of AlDoped $\mathrm{ZnO}$ via Phosphonic Acid-Anchored C60 SAMs, Advanced Energy Materials 2 (2012) 532-535.

[10] W. Tress, K. Leo, M. Riede, Influence of Hole-Transport Layers and Donor Materials on Open-Circuit Voltage and Shape of I-V Curves of Organic Solar Cells, Advanced Functional Materials 21 (2011) 2140-2149.

[11] W. Tress, A. Petrich, M. Hummert, M. Hein, K. Leo, M. Riede, Imbalanced mobilities causing S-shaped IV curves in planar heterojunction organic solar cells, Applied Physics Letters 98 (2011) 063301.

[12] W. Tress, S. Pfuetzner, K. Leo, M. Riede, Open circuit voltage and IV curve shape of $\mathrm{ZnPc:C60}$ solar cells with varied mixing ratio and hole transport layer, Journal of Photonics for Energy 1 (2011) 011114.

[13] A. Kumar, S. Sista, Y. Yang, Dipole induced anomalous S-shape I-V curves in polymer solar cells, Journal of Applied Physics 105 (2009) 094512.

[14] J. Wang, X. Ren, S. Shi, C. Leung, P. K. Chan, Charge accumulation induced S-shape JV curves in bilayer heterojunction organic solar cells, Organic Electronics 12 (2011) 880-885.

[15] J. Wagner, M. Gruber, A. Wilke, Y. Tanaka, K. Topczak, A. Steindamm, U. Hormann, A. Opitz, Y. Nakayama, H. Ishii, J. Pflaum, N. Koch, W. Brutting, Identification of different origins for s-shaped current voltage characteristics in planar heterojunction organic solar cells, Journal of Applied Physics 111 (2012) 054509.

[16] B. Ecker, H.-J. Egelhaaf, R. Steim, J. Parisi, E. von Hauff, Understanding S-Shaped Current-Voltage Characteristics in Organic Solar Cells Containing a TiOx Interlayer with Impedance Spectroscopy and Equivalent Circuit Analysis, The Journal of Physical Chemistry C 116 (2012) 16333-16337.

[17] W. Tress, Device physics of organic solar cells, Dissertation, TU Dresden (2011).

[18] W. Tress, S. Corvers, K. Leo, M. Riede, Investigation of Driving Forces for Charge Extraction in Organic Solar Cells: Transient Photocurrent Measurements on Solar Cells Showing S-Shaped Current-Voltage Characteristics, Advanced Energy Materials (2013) n/a-n/a.

[19] E. Wang, L. Hou, Z. Wang, S. Hellström, F. Zhang, O. Inganäs, M. R. Andersson, An easily synthesized blue polymer for highperformance polymer solar cells., Advanced Materials 22 (2010) 5240-4.

[20] Z. Tang, L. M. Andersson, Z. George, K. Vandewal, K. Tvingstedt, P. Heriksson, R. Kroon, M. R. Andersson, O. Inganäs, Interlayer for modified cathode in highly efficient inverted ITOfree organic solar cells., Advanced Materials 24 (2012) 554-8.

[21] M. Glatthaar, M. Niggemann, B. Zimmermann, P. Lewer, M. Riede, A. Hinsch, J. Luther, Organic solar cells using inverted layer sequence, Thin Solid Films 491 (2005) 298-300.

[22] H. B. Michaelson, The work function of the elements and its periodicity, Journal of Applied Physics 48 (1977) 4729.

[23] A. M. Goodman, Photoemission of Holes and Electrons from Aluminum into Aluminum Oxide, Journal of Applied Physics 41 (1970) 2176.

[24] M. Schaer, F. Nüesch, D. Berner, W. Leo, L. Zuppiroli, Water Vapor and Oxygen Degradation Mechanisms in Organic Light Emitting Diodes, Advanced Functional Materials 11 (2001) $116-121$.

[25] K. Norrman, S. A. Gevorgyan, F. C. Krebs, Water-induced degradation of polymer solar cells studied by H2(18)O labeling., ACS applied materials \& interfaces 1 (2009) 102-12.

[26] Y. Hirose, A. Kahn, V. Aristov, P. Soukiassian, V. Bulovic S. R. Forrest, Chemistry and electronic properties of metalorganic semiconductor interfaces: $\mathrm{Al}, \mathrm{Ti}, \mathrm{In}, \mathrm{Sn}, \mathrm{Ag}$, and $\mathrm{Au}$ on PTCDA., Physical Review B 54 (1996) 13748-13758.

[27] J. Y. Kim, S. H. Kim, H.-H. Lee, K. Lee, W. Ma, X. Gong,
A. J. Heeger, New Architecture for High-Efficiency Polymer Photovoltaic Cells Using Solution-Based Titanium Oxide as an Optical Spacer, Advanced Materials 18 (2006) 572-576.

[28] W. Tress, Z. Tang, K. Tvingstedt, O. Inganäs, Plasma treatment of active layer to increase adhesion, in preparation (????). 